\title{
Problemas socioambientales y educación ambiental. El cambio climático desde la perspectiva de los futuros maestros de educación primaria
}

\author{
Socio-Environmental Problems and Environmental \\ Education. Climate Change from the Perspective of \\ Future Elementary School Teachers
}

\section{Olga Moreno-Fernández}

Universidad de Sevilla

\section{Resumen}

El cambio climático ha cobrado gran relevancia en los últimos años a nivel mundial. Esta problemática socioambiental ha reactivado la política ambiental tras el movimiento "Viernes para el futuro", liderado por la activista Greta Thunberg y puso de manifiesto la relevancia que tiene la temática. El objetivo de esta investigación fue conocer las concepciones de los futuros maestros de educación primaria acerca del cambio climático y la necesidad de su tratamiento en las escuelas. Se realizó un estudio descriptivo-exploratorio en el que participaron 261 maestros y maestras en formación inicial del Grado de Educación Primaria de la Universidad de Sevilla (Espańa). Los datos se recogieron a través de un cuestionario estructurado de 11 preguntas. Los resultados destacan que la noción de cambio climático se asocia con extinción y destrucción, así como también se evidencia un reconocimiento mayoritario de la causalidad humana en el origen del problema y una necesidad inminente de abordarlo educativamente desde las aulas escolares.

Palabras clave: cambio climático, educación ambiental, educación primaria, maestros en formación inicial, problemas socioambientales.

\section{Correspondencia a:}

Olga Moreno-Fernández

Departamento de Didáctica de las Ciencias Experimentales y Sociales, Facultad de Educación, Universidad de Sevilla. Calle Pirotecnia s/n, Despacho 4.81. 41013 Sevilla, España.

omoreno@us.es

(C) 2020 PEL, http://www.pensamientoeducativo.org - http://www.pel.cl 


\section{Abstract}

Climate change has become very important worldwide in recent years. This socioenvironmental problem has reactivated environmental policy in the wake of the "Friday for the Future" movement, led by activist Greta Thunberg, and highlighted the importance of the issue. The aim of this research was to find out the conceptions of future elementary school teachers about climate change and the need to address it in schools. A descriptiveexploratory study was carried out in which 261 teachers in initial training in the Elementary Education Degree at the University of Seville (Spain) participated. The data were collected through a structured questionnaire containing 11 questions. The results highlight that the notion of climate change is associated with extinction and destruction, as well a majority of participants recognizing human causality in the origin of the problem and an imminent need to address it in educational terms in school classrooms.

Keywords: climate change, environmental education, elementary education, socio-environmental problems, teachers in initial training.

\section{Introducción}

El cambio climático ha cobrado gran preeminencia en los últimos ańos a nivel mundial, reactivándose las políticas ambientales tras el movimiento "Viernes para el futuro", liderado por la activista Greta Thunberg, lo cual pone de manifiesto la relevancia que tiene la educación ambiental. Y es que el cambio climático es, sin duda, uno de los mayores problemas socioambientales a los que se enfrenta hoy la humanidad, no solo por la diversidad de factores que lo provocan y las consecuencias que genera, sino por su dimensión planetaria, lo que hace que sea una cuestión de gran complejidad (Bangay \& Blum, 2010; González-Gaudiano y MeiraCartea, 2020; Reis \& Ballinger, 2020). En efecto, se trata de una problemática que no solo pone en peligro la vida tal y como la conocemos, sino que además es el centro de las graves complicaciones sociales, ambientales y económicas actuales, lo que afecta la totalidad de las actividades humanas (Schewe et al., 2019). Dada su complejidad, para combatirlo la sociedad debiera generar respuestas múltiples que provengan desde varios sectores, entre ellos del ámbito educativo.

\section{Estado de la cuestión}

A nivel internacional, la Convención Marco de las Naciones Unidas sobre el cambio climático en 1992 proporcionó una base para la acción, poniendo de manifiesto en su artículo 6 la necesidad de que la comunidad internacional centrara sus esfuerzos en la educación, capacitación, empoderamiento y compromiso socioambiental de nuestro mundo. Posteriormente, el Acuerdo de París, en su artículo 12, animó a las naciones a "mejorar la educación, la capacitación, la sensibilización, la participación y el acceso a la información sobre el cambio climático" (Convención Marco de las Naciones Unidas sobre el Cambio Climático, 2015). En apoyo de estas iniciativas, Action for Climate Empowerment (ACE), trabaja por concienciar en torno al cambio climático y la Educación para el Desarrollo Sostenible (ESD) a nivel internacional, promoviendo la adquisición de conocimientos, habilidades y actitudes apropiados para empoderar a las comunidades y que contribuyan a la lucha contra los problemas socioambientales a los que nos enfrentamos, abordando para ello los Objetivos de Desarrollo 
Sostenible 4.7 y 13.3 que forman parte de la Agenda para el Desarrollo Sostenible de 2030 (United Nations, UN, 2015). Desde esta perspectiva, concebimos la educación ambiental como lo señalado por Moreno-Fernández y Navarro-Díaz (2015), esto es, como un

proceso a través del cual deben facilitarse a la ciudadanía aquellos mecanismos necesarios para la concienciación y sensibilización ante los problemas socioambientales que nos rodean, de tal manera que la relación individuo-sociedad-entorno se transforme, se reinvente, en la debida relación de respeto mutuo y habitabilidad, entendida esta última en parámetros cualitativos, de calidad de vida (p. 38).

Para ello, es necesario reflexionar acerca de las actitudes y valores que comprende la educación ambiental, favoreciendo aquellas experiencias de aprendizaje que promuevan la participación activa en la búsqueda de soluciones integrales a los problemas actuales, puesto que estos van cambiando y pueden variar a lo largo de los años. Por tanto, la educación ambiental no solo debe dotar de valores y habilidades, sino también desarrollar "competencias para actuar con criterios de sostenibilidad; es decir, con la capacidad de utilizar los conocimientos y habilidades en otros contextos, tanto a nivel individual como colectivo" (Álvarez y Vega, 2009, p. 254), sin olvidar profundizar en las dimensiones emocionales de la educación ambiental (Russell, 2016).

Por tanto, podemos afirmar que la educación juega un papel esencial, donde sin duda, y como seńalan Bangay y Blum (2010),

la integración del aprendizaje sobre el medio ambiente de calidad en los sistemas educativos existentes representa un desafío tanto inmediato como a largo plazo para responder al cambio climático. El desafío inmediato es el de los sistemas educativos a prueba de clima (adaptación), mientras que la tarea más larga y difícil es la de desarrollar sistemas educativos que doten a los alumnos de las actitudes, los conocimientos y los atributos necesarios para hacer frente a los desafíos futuros (p. 364).

En este sentido, el papel de las instituciones de educación superior ante el cambio climático es de gran importancia para hacer frente a los desafíos a los que nos enfrentamos, ya que es en las universidades donde se forma, entre otros muchos profesionales, a los futuros profesores que trabajarán con las nuevas generaciones en las aulas escolares (Karatzoglou, 2013; Leal-Filho, 2010; Leal-Filho et al., 2018; Molthan-Hill, Worsfold, Nagy, Leal-Filho \& Mifsud, 2019). Sin embargo, y a pesar de la relevancia que tiene trabajar desde el ámbito universitario cuestiones relacionadas con la educación ambiental, las investigaciones realizadas al respecto ponen de manifiesto que gran parte del profesorado universitario aún no ha incorporado estos temas a su práctica docente (Azcárate, Navarrete y García, 2012; Aznar, Martínez-Agut, Palacios, Piñero \& Ull, 2011), siendo imprescindible por parte de la comunidad universitaria integrar la educación ambiental como una prioridad central en la alfabetización básica de los docentes de todos los niveles educativos (Solís-Espallargas y Valderrama-Hernández, 2015). Para ello es necesario que se incluyan en las materias universitarias contenidos relacionados con las problemáticas socioambientales de nuestro mundo, abordando estas cuestiones interdisciplinarmente, de modo de superar la dualidad clásica entre lo social y lo natural. De igual manera, debe concienciarse al profesorado de la importancia y la responsabilidad de trabajar estos contenidos a nivel conceptual, procedimental y actitudinal, creando espacios de reflexión y colaboración en los que pensar críticamente, tanto individual como colectivamente, en cuanto a la relevancia de una praxis educativa responsable.

En este sentido, uno de los obstáculos detectados en el ámbito universitario es la falta de atención en torno a estas problemáticas, lo que lleva a que el tratamiento que se hace de ellas sea sesgado y reduccionista (Barrón, Navarrete y Ferrer-Balas, 2010; García-Díaz, 2004); por ello, urge "una educación que ayude a los individuos a interpretar, comprender y conocer la complejidad y globalidad de los problemas que se producen en el mundo y enseñe actitudes, conocimientos, valores, comportamientos, etc.” (Álvarez y Vega, 2009, p. 246). 
De este modo la institución universitaria, como responsable de la formación del futuro profesorado, se debe socialmente al compromiso de superar esa división entre lo social y lo natural, y "buscar nuevos planteamientos educativos que nos sitúen en una realidad donde las cuestiones ambientales son, en gran medida, cuestiones sociales" (Solís-Espallargas y Valderrama-Hernández, 2015, p. 169), siendo perentorio regenerar las formas en que vivimos en esta tierra (McKenzie, Hart, Bai \& Jickling, 2009).

Según investigaciones realizadas (Escoz-Roldán, Gutiérrez-Pérez, Arto-Blanco y Meira-Cartea, 2017; MeiraCartea, Gutiérrez-Pérez, Arto-Blanco, Escoz-Rodán, 2018), a pesar de que las experiencias en educación en torno al cambio climático no han sido del todo satisfactorias como mecanismo de adaptación, mitigación, concienciación e implicación ciudadana, no hay duda de que la educación juega un papel primordial en el tratamiento de este complejo asunto, por lo que se debe seguir trabajando hacia el objetivo de formar ciudadanos concienciados, informados, e implicados con el cambio climático (Bangay \& Blum, 2010; Mochizuki \& Bryan, 2015).

Uno de los mayores inconvenientes de enseñar la relevancia y la urgencia que supone el problema del cambio climático es el nivel de abstracción del fenómeno en sí mismo, una idea que queda muy bien expresada por Giddens (2010) cuando afirma que "como los peligros que representa el calentamiento global no son tangibles, inmediatos, ni visibles en el curso de la vida cotidiana, por muy formidables que puedan parecer, muchos se cruzarán de brazos y no harán nada al respecto" (p. 12). Y es que superar la desconexión que se produce entre la preocupación por los problemas socioambientales e incorporar en nuestra vida cotidiana conductas sostenibles sigue siendo uno de los grandes retos de la educación ambiental. Aunque son varias las cuestiones que generan esa desconexión entre lo que se piensa y la manera en que se actúa, es preciso reconocer que las aportaciones - por pequeñas que sean - nunca son insignificantes y siempre agregan valor a la resolución del problema. De esta forma, podemos señalar que una de las principales barreras para no cambiar de hábitos está muy relacionada, precisamente, con lo que hacemos desde nosotros mismos, lo que puede parecer poco representativo e insignificante, o bien, que no va a repercutir en un cambio lo suficientemente relevante para que merezca el esfuerzo, derivando a la larga en un distanciamiento de la problemática a la que nos enfrentamos.

Se trata, pues, de un alejamiento del problema que no ha estado ajeno al ámbito educativo y que, a pesar del gran número de estudios que han profundizado en cuestiones relacionadas con el cambio climático — tanto desde la perspectiva del currículo, del alumnado y en menor medida del profesorado, además de diversos niveles educativos-, estos siguen evidenciando el desconocimiento existente respecto de este fenómeno (Barbosa, Lima \& Machado, 2012; Barros y Pinheiro, 2013; Escoz-Roldán et al., 2017; García-Rodeja y De Oliveira, 2012; Hess \& Collins, 2018; Hess \& Maki, 2019; Meira-Cartea et al., 2018; Molthan-Hill et al., 2019; Reis \& Ballinger, 2020; Sternäng \& Lundholm, 2011).

En este sentido es más que necesario seguir trabajando desde la educación, tanto a nivel formal como no formal, y desde la educación infantil hasta la educación superior, ya que esta juega un importante papel para hacer frente a un cambio que hoy es más necesario que nunca. Ello, ya que la función de la educación es capacitar al alumnado para desarrollar los conocimientos, actitudes y capacidades que le permitan pensar de manera crítica, resolver problemas y encarar la incertidumbre, para lo cual se debe contar con una visión holística de esta problemática.

Los sistemas educativos, por su parte, enfrentan diversos retos, donde el más inmediato es adaptarse a trabajar el cambio climático y el más largo y difícil es desarrollar metodologías educativas que provean al alumnado de los conocimientos, habilidades y capacidades necesarias para hacer frente a los desafíos futuros (Bangay \& Blum, 2010).

Se trata, pues, de un escenario en el que sin duda el profesorado ocupa un papel primordial, por lo que es necesario investigar cómo estos profesionales en formación inicial y aquellos que todavía se están formando para estar a corto o mediano plazo trabajando en las aulas, entienden el cambio climático y sus diferentes factores, 
con la finalidad de reorientar su formación hacia una más ajustada a las necesidades actuales y que, en su futura labor docente, promueva que su alumnado adquiera las herramientas necesarias para afrontar cuestiones socioambientales tan relevantes como el cambio climático.

De esta forma, estas relevantes cuestiones llevaron a los siguientes objetivos de investigación del presente estudio:

- Conocer qué entiende el profesorado en formación inicial de educación primaria por cambio climático.

- Explorar los conocimientos que el profesorado en formación inicial de educación primaria tiene respecto de las causas, consecuencias y estrategias que se pueden llevar a cabo para mitigar el cambio climático.

- Determinar la relevancia que tiene el cambio climático como problemática socioambiental a tratar en educación primaria, desde la perspectiva del futuro profesorado especializado en este nivel educativo.

\section{Métodos y materiales}

Se realizó un estudio descriptivo e interpretativo a través de la utilización de instrumentos como el cuestionario de preguntas abiertas, combinando el enfoque cualitativo básico con el apoyo cuantitativo para el tratamiento de datos, así como técnicas de "análisis del contenido" (Cohen y Manion, 2002).

\section{Participantes}

Se realizó un muestreo intencional y de conveniencia en donde participaron 261 profesores en formación inicial de educación primaria, es decir, alumnos del Grado de Educación Primaria de la Universidad de Sevilla. De ellos, el 67,4\% $(n=176)$ eran mujeres y el 32,6\% $(n=85)$ hombres. Las edades de los participantes estaban comprendidas entre los 19 y 22 años.

\section{Instrumento}

Se elaboró un cuestionario ad hoc para explorar los conocimientos de los participantes de la investigación. El instrumento inicial tomó como base el utilizado por Meira-Cartea y Arto-Blanco (2014), mientras que la versión final fue revisada por dos expertas en cambio climático, con lo cual el instrumento quedó conformado por 11 preguntas y 5 dimensiones: Percepción sobre el cambio climático; Causas del cambio climático; Consecuencias del cambio climático; Estrategias para mitigar el cambio climático; y por último, Educación y cambio climático (Tabla 1).

Tabla 1

Categorias y preguntas del instrumento

Categorías Preguntas

\begin{tabular}{|c|c|}
\hline Percepción & $\begin{array}{l}\text { 1. ¿Cuál es el primer pensamiento o imagen que te viene a la cabeza cuando escuchas } \\
\text { hablar del cambio climático? }\end{array}$ \\
\hline \multirow{3}{*}{ Causas } & 2. ¿Cuál piensas que es la principal causa del cambio climático? \\
\hline & $\begin{array}{l}\text { 3. ¿Piensas que el cambio climático se debe a la intervención humana, a cuestiones } \\
\text { puramente naturales, o a una combinación de ambas? }\end{array}$ \\
\hline & $\begin{array}{l}\text { 4. ¿Crees que el cambio climático está relacionado con otros problemas } \\
\text { socioambientales? ¿Cuáles? }\end{array}$ \\
\hline Consecuencias & $\begin{array}{l}\text { 5. ¿Qué consecuencias crees que tiene el cambio climático para el planeta? ¿Cómo } \\
\text { nos afecta? }\end{array}$ \\
\hline
\end{tabular}




\begin{tabular}{|c|c|}
\hline Estrategias & $\begin{array}{l}\text { 6. ¿Qué estrategias crees que se pueden llevar a cabo para mitigar el cambio } \\
\text { climático? }\end{array}$ \\
\hline \multirow{5}{*}{ Educación } & 7. ¿Crees que es necesario abordar el cambio climático en las aulas de primaria? \\
\hline & $\begin{array}{l}\text { 8. Si has contestado afirmativamente a la pregunta anterior ¿Dentro de qué materia o } \\
\text { materias lo abordarías? Marca aquellas que consideres oportunas. }\end{array}$ \\
\hline & $\begin{array}{l}\text { 9. Si nos remitimos a la legislación educativa vigente ¿dentro de qué materia o } \\
\text { materias consideras que se aborda el cambio climático? }\end{array}$ \\
\hline & $\begin{array}{l}\text { 10. Cómo futuro/a maestro/a de educación primaria que serás ¿Consideras que estás } \\
\text { capacitado/a para tratar este tema con el alumnado? }\end{array}$ \\
\hline & 11. ¿Crees que estás lo suficientemente formado/a para tratar el tema? \\
\hline
\end{tabular}

Fuente: Elaboración propia a partir de las aportaciones de Meira-Cartea y Arto-Blanco (2014).

El cuestionario se aplicó en las aulas entre septiembre de 2019 y febrero de 2020, para lo cual se acudió a cada una de las aulas en las que interesaba recoger datos. Se explicó al alumnado la finalidad de la investigación y se informó respecto del anonimato de sus datos, así como de la participación totalmente voluntaria en el estudio.

\section{Resultados}

\section{Percepción del cambio climático}

Para la pregunta 1. ¿Cuál es el primer pensamiento o imagen que te viene a la cabeza cuando escuchas hablar del cambio climático?, se siguió el sistema de clasificación de Meira-Cartea y Arto-Blanco (2014), agrupándose las respuestas en 12 categorías (Tabla 2).

Tabla 2

Primer pensamiento o imagen acerca del cambio climático

\begin{tabular}{lcc}
\multicolumn{1}{c}{ Primer pensamiento o imagen } & Frecuencia & Porcentaje \\
\hline Deshielo de los polos/glaciares & 39 & $14,9 \%$ \\
\hline Contaminación & 32 & $12,3 \%$ \\
\hline Aumento de las temperaturas & 29 & $11,1 \%$ \\
\hline Destrucción & 27 & $10,3 \%$ \\
\hline Desastres naturales & 25 & $9,6 \%$ \\
\hline Efecto invernadero & 23 & $8,8 \%$ \\
\hline Pérdida de biodiversidad & 21 & $8,0 \%$ \\
\hline Extinción humana & 19 & $7,3 \%$ \\
\hline Falta de conciencia/irresponsabilidad & 16 & $6,1 \%$ \\
\hline Fenómenos meteorológicos & 11 & $4,2 \%$ \\
\hline Fenómenos naturales & 9 & $3,4 \%$ \\
\hline Futuro (concienciación/esfuerzo) & 7 & $2,7 \%$ \\
\hline Otros & 3 & $1,1 \%$ \\
\hline
\end{tabular}

Fuente: Elaboración propia. 
Como se aprecia, cuando el profesorado en formación inicial de educación primaria piensa en el cambio climático lo relaciona principalmente con el deshielo de los glaciares y, por lo tanto, de los polos (14,9\%), a la contaminación $(12,3 \%)$ y al aumento de las temperaturas $(11,1 \%)$. Las consecuencias son las más nombradas por el profesorado en formación inicial cuando piensan en el cambio climático (74,2\%), mientras que las causas solo son reflejadas en un $18,4 \%$ de los casos. Los datos coinciden con estudios anteriores, tanto nacionales como internacionales (Leiserowitz, 2004; 2007; Meira-Cartea, 2013; Meira-Cartea y Arto-Blanco, 2014;). Solo el 3,4\% señaló una causalidad natural del fenómeno y un 4,2\% lo relacionó con fenómenos meteorológicos. El 1,1\% hizo mención de otras cuestiones.

\section{Causas del cambio climático}

El profesorado en formación inicial participante del estudio consideró que la principal causa del cambio climático era la incidencia del ser humano sobre el planeta, lo cual repercute en cuestiones relativas a la contaminación, los residuos o el mal uso de los recursos naturales, tal y como se ejemplifica en las siguientes unidades de información:

- C17: Los residuos perjudiciales que afectan a la capa de ozono producidos por el hombre, las fábricas, los desechos, etc.

- C61: El excesivo consumo y la explotación de los recursos por parte de los seres humanos, especialmente de las fábricas.

- C136: La mala acción del hombre, el mal uso de los nuevos desarrollos industriales y tecnológicos, es evidente que debemos evolucionar, pero siempre cuidando aquello que nos permite la vida, la naturaleza.

En cuanto a si el cambio climático se debe a la intervención humana, a cuestiones puramente naturales, o a una combinación de ambas, el $62,8 \%$ del profesorado en formación inicial $(n=164)$ señaló la intervención humana exclusivamente, mientras que el 37,2\% $(n=97)$ indicó que este se debe a una combinación de factores naturales junto con la actividad humana, aunque influiría más esta última, tal y como se ejemplifica en las siguientes unidades de información:

- C27: Combinación de ambas, pero la aceleración se debe al ser humano.

- C83: Una combinación de ambas, aunque mucho más por la intervención humana.

- C101: Principalmente intervención humana, aunque la naturaleza influye. Yo diría que es una combinación de ambas.

Así mismo, un 93,5\% $(n=244)$ del profesorado en formación inicial consideró que el cambio climático estaba relacionado con otros problemas socioambientales y un $6,5 \%(n=17)$ señaló que esto no era efectivo. A los participantes que contestaron afirmativamente se les solicitó que mencionaran cuáles eran esos problemas concretos a los que asociaban con el cambio climático. El 43,7\% $(n=114)$ hizo referencia a diversas cuestiones que se agruparon en la categoría de procesos biofísicos, concentrando situaciones de deterioro ambiental relacionadas con actividades humanas principalmente contaminantes, siendo esta clase la que convocó más respuesta. Por su parte, el 24,5\% $(n=64)$ señaló problemas socioambientales, mientras que el 19,5\% $(n=51)$ lo relacionó con cuestiones referidas a la falta de educación y un 5\% $(n=13)$ a fenómenos catastróficos. Otro $5 \%(n=13)$ se recogió en la categoría otros. Por último, la categoría con un menor índice de respuesta fue la de fenómenos terrestres, con un $2,4 \%(n=6)$ de las respuestas recogidas.

Al comparar los resultados para esta pregunta y el tipo de causas que provoca el cambio climático expresadas por los participantes, los datos coinciden en la prevalencia de orígenes relacionados con la incidencia de la actividad humana en el medio. Ello es consistente con otros estudios, donde como señalan Meira-Cartea y Arto-Blanco (2014): 
las lógicas explicativas que aparecen en la categoría de fenómenos terrestres se vinculan, no con los casos que aluden a una causalidad natural del cambio climático, sino con aquellos que reconocen un peso relativamente importante, pero minoritario, de las causas naturales (p. 24).

\section{Consecuencias del cambio climático}

En relación con las consecuencias derivadas del cambio climático, todos los participantes del estudio señalaron que las ramificaciones del problema eran negativas. Así mismo, puesto que la interrogante se les planteó como pregunta abierta, los participantes podían enunciar una o más de una consecuencia, de modo que las respuestas se agruparon en un total de 10 ítems con 806 respuestas (Tabla 3).

Tabla 3

Consecuencias del cambio climático

Consecuencias del cambio climático

Frecuencia Porcentaje

\begin{tabular}{lcc}
\hline Aumento de las temperaturas/olas de calor más intensas & 196 & $75,0 \%$ \\
\hline Afectación en la vida de las personas tal y como la conocemos & 102 & $39,0 \%$ \\
\hline Aumento del nivel del mar & 96 & $36,7 \%$ \\
\hline Cambio de los ecosistemas & 82 & $31,4 \%$ \\
\hline Pérdida de la biodiversidad & 80 & $30,6 \%$ \\
\hline $\begin{array}{l}\text { Desastres naturales de mayor intensidad (huracanes, tsunamis, } \\
\text { tormentas más intensas; inundaciones, sequías) }\end{array}$ & 66 & $25,2 \%$ \\
\hline Propagación de enfermedades & 65 & $24,9 \%$ \\
\hline Derretimiento de los glaciares/deshielo de los polos & 55 & $21,0 \%$ \\
\hline Contaminación (aumento $\mathrm{CO}_{2}$ atmósfera) & 49 & $18,7 \%$ \\
\hline Alimentos más caros & 16 & $6,1 \%$ \\
\hline
\end{tabular}

Fuente: Elaboración propia.

\section{Estrategias para mitigar el cambio climático}

Entre las estrategias que el profesorado en formación inicial consideró que se podían llevar a cabo para mitigar el cambio climático, la principal propuesta fue la necesidad de cambiar el estilo de vida que tenemos para transitar hacia modelos menos consumistas y más responsables con los recursos, lo cual quedó de manifiesto en el 23,4\% $(n=61)$ de los casos. En segundo lugar, el $16,8 \%(n=44)$ propuso un cambio a energías renovables y un menor uso de combustibles fósiles, aspecto que se recoge en los informes del Grupo Intergubernamental de Expertos sobre el Cambio Climático (IPCC, por sus siglas en inglés) (2013; 2018). La tercera opción fue señalada por el $16,2 \%(n=42)$, que concordó en la importancia de reflexionar, tomar conciencia y favorecer la educación en este tipo de cuestiones. Como cuarta opción, el 13,4\% $(n=35)$ seńaló la necesidad de reducir las emisiones para bajar el grado de contaminación del aire, seguido por el 10\% $(n=26)$ que hizo referencia a la industria. Reciclar, reutilizar, reducir residuos se situó en la sexta opción, junto con la propuesta de elaboración de leyes y acuerdos internacionales, con un 6,5\% $(n=17)$ cada una de ellas. Solo un 3,4\% $(n=9)$ de los participantes consideró que no había solución para frenar el cambio climático, aduciendo que es demasiado tarde. Por último, el 3,8\% $(n=10)$ contestó otras cuestiones (Tabla 4). Estas estrategias coinciden con las propuestas por futuros profesionales de la educación, aunque no en el mismo orden de importancia (Meira-Cartea, 2013; Meira-Cartea y Arto-Blanco, 2014). 
Tabla 4

Estrategias para mitigar del cambio climático

\begin{tabular}{lcc}
\multicolumn{1}{c}{ Estrategias } & Frecuencia & Porcentaje \\
\hline $\begin{array}{l}\text { Cambio en los estilos de vida (nuevo modelo de producción menos } \\
\text { consumista). Consumo responsable de los recursos. }\end{array}$ & 61 & $23,4 \%$ \\
\hline $\begin{array}{l}\text { Cambio a energías renovables y un menor uso de combustibles } \\
\text { fósiles. }\end{array}$ & 44 & $16,8 \%$ \\
\hline Reflexionar, tomar conciencia. Educación. & 42 & $16,2 \%$ \\
\hline No contaminar, menos emisiones. Contaminación del aire. & 35 & $13,4 \%$ \\
\hline $\begin{array}{l}\text { Industrias (reducir contaminación, aplicar impuestos, cierre de } \\
\text { centros). }\end{array}$ & 26 & $10,0 \%$ \\
\hline Reciclar, reutilizar, reducir residuos. & 17 & $6,5 \%$ \\
\hline Leyes. Acuerdos internacionales. & 17 & $6,5 \%$ \\
\hline Demasiado tarde para hacer frente al cambio climático. & 9 & $3,4 \%$ \\
\hline Otros. & 10 & $3,8 \%$ \\
\hline
\end{tabular}

Fuente: Elaboración propia.

\section{Cambio climático y educación}

Respecto de la cuestión ¿Crees que es necesario abordar el cambio climático en las aulas de primaria?, el 100\% de los participantes contestó afirmativamente. Para profundizar en las respuestas obtenidas se optó por clasificarlas siguiendo una hipótesis de progresión, es decir, ordenando las respuestas de lo más simple a lo más complejo (Tabla 5).

Tabla 5

Necesidad de abordar el cambio climático en las aulas de primaria

Nivel

Descripción del nivel

Frecuencia Porcentaje

\begin{tabular}{|c|c|c|c|}
\hline Nivel & Descripción del nivel & Frecuencia & Porcentaje \\
\hline $\begin{array}{l}\text { Cosmovisión } \\
\text { sincrética (nivel } \\
\text { básico). }\end{array}$ & $\begin{array}{l}\text { Los participantes se limitan a seńalar que es } \\
\text { necesario o imprescindible tratar este tema } \\
\text { con el alumnado de primaria, pero sin entrar } \\
\text { en detalles. }\end{array}$ & 130 & $49,9 \%$ \\
\hline $\begin{array}{l}\text { Cosmovisión analítica } \\
\text { (nivel intermedio). }\end{array}$ & $\begin{array}{l}\text { Los participantes, además de estar de acuerdo } \\
\text { con que es una temática necesaria de abordar } \\
\text { en educación primaria, señalan la necesidad } \\
\text { de dotar al alumnado de herramientas que } \\
\text { le permita entender el cambio climático, así } \\
\text { como sus causas y consecuencias. }\end{array}$ & 33 & $12,6 \%$ \\
\hline $\begin{array}{l}\text { Aproximación a una } \\
\text { cosmovisión sistémica } \\
\text { (aproximación a } \\
\text { un conocimiento } \\
\text { deseable). }\end{array}$ & $\begin{array}{l}\text { Los participantes no solo consideran que es } \\
\text { necesario y relevante dotar de herramientas } \\
\text { adecuadas al alumnado, sino que van más } \\
\text { allá al mencionar la importancia que tiene } \\
\text { trabajar estos contenidos en la formación } \\
\text { de quienes van a ser los futuros ciudadanos } \\
\text { y la necesidad de que estén concienciados } \\
\text { respecto de un tema tan sensible } \\
\text { ambientalmente como es el cambio climático. }\end{array}$ & 98 & $37,5 \%$ \\
\hline
\end{tabular}

Fuente: Elaboración propia. 
De esta forma, $49,9 \%(\mathrm{n}=130)$ respondió simplemente con afirmaciones del tipo C1: "Sí, muy necesario" o C16: "Claro, muy necesario, imprescindible". El 12,6\% ( $\mathrm{n}=33)$, además de estar de acuerdo con que se trata de una temática necesaria de abordar en educación primaria, hizo mención de la importancia de ir más allá de lo que habitualmente viene en los libros de texto, dotando al alumnado de herramientas para poder entender el cambio climático, sus causas y consecuencias. Ejemplo de esto se extrae de unidades de información como C125: "Sí, y que sea llevado a la realidad, es decir, que no se quede meramente mencionado por los libros" o C238: "Sí, debemos darle herramientas para poder entenderlo y combatirlo". Por último, el 37,5\% (n = 98) señaló la importancia de trabajar estos contenidos en la formación de quienes van a ser los futuros ciudadanos y la necesidad de que estén concienciados acerca del tema. Esto se extrae de unidades de información como las siguientes:

- C10: Por supuesto, pero no de forma rápida, sino haciendo hincapié, debido a la importancia que tiene. El alumnado debe ser consciente de las repercusiones y futuro que les espera, ellos pueden ser el arma más potente contra esta desfavorable situación.

- C112: Sí, puesto que son el futuro de nuestro mundo; si educamos a los alumnos de forma correcta en el cuidado del medioambiente tendrán en su conciencia la mayoría de los aspectos necesarios para cuidarlo.

- C260: Sí, puesto que en primaria formamos a los futuros ciudadanos, y si se conciencian y llegan a entender la importancia que tiene desde pequeños, será más fácil ir afianzando esos pensamientos y que ayuden de una manera activa.

En relación con la pregunta acerca de cuál materia o materias tratarías el cambio climático en el aula de educación primaria, el 62,5\% $(n=163)$ señaló la asignatura de Ciencias de la Naturaleza, seguido del 31\% $(n=$ 81) que priorizó la asignatura de Ciencias Sociales. En tercer lugar, el 6,1\% $(n=16)$ escogió Educación en Valores/ Educación para la Ciudadanía. Solo el 0,4\% $(n=1)$ consignó que el tema debía abordarse transversalmente en todas las asignaturas de la etapa de primaria ${ }^{1}$.

Los datos recopilados ponen de relieve una dualidad entre las Ciencias Naturales y las Ciencias Sociales que se ha venido realizando habitualmente y que pierde todo su sentido si la intención es explicar y abarcar las problemáticas actuales en toda su complejidad. Sin embargo, otros estudios con profesorado en formación inicial, como el de López-Fernández y Oller-Freixa (2019), señalan que este tipo de problemas socioambientales se siguen abordando desde la perspectiva de las Ciencias de la Naturaleza. Esto puede deberse a que el currículo para la etapa de educación primaria tradicionalmente ha dividido estos contenidos entre las asignaturas de Ciencias de la Naturaleza y Ciencias Sociales. Solo la Ley Orgánica de Educación, LOE (2006), vigente en España de 2006 a 2013, consiguió hacerlas coincidir en la asignatura denominada Conocimiento del Medio Natural, Social y Cultural.

$\mathrm{Al}$ respecto, una forma de entender las problemáticas socioambientales en general, y el cambio climático en particular, es que hablamos de un proceso complejo en el que intervienen factores ambientales y factores naturales, por lo que consideramos que debe abordarse desde ambas disciplinas, conjuntamente.

Ahora bien, si planteamos al profesorado en formación inicial dentro de qué materia o materias consideran que la legislación educativa vigente aborda el cambio climático, las respuestas se polarizan aún más entre la asignatura de Ciencias de la Naturaleza y la de Ciencias Sociales. En este sentido, el 62,9\% ( $n=164)$ señaló que el currículo aborda el cambio climático en la asignatura de Ciencias de la Naturaleza; el 34,5\% $(n=90)$ puntualizó que en la asignatura de Ciencias Sociales, y un 2,6\% $(n=7)$ declaró no tenerlo claro y no poder decantarse. Sin embargo, si nos remitimos al currículo vigente, el RD 126/2014 establece que el cambio climático es un contenido a tratar en la materia de Ciencias Sociales, concretamente en su "Bloque 2. El mundo en que vivimos", en el cual se hace mención al desarrollo sostenible, a los problemas de la contaminación, a las causas y consecuencias del cambio

1. Esto comprendería las siguientes asignaturas: Matemáticas, Lengua Castellana y Literatura, Lengua Extranjera, Educación Artística y plástica, Educación Física o Religión. 
climático y al consumo responsable. Desde esta perspectiva, entonces, este desconocimiento del currículo por parte del profesorado en formación inicial puede incidir en que no se aborde la temática desde una perspectiva compleja y acorde a sus características.

En cuanto a la instrucción que tiene el profesorado en formación inicial en torno al tema del cambio climático, el 87,4\% $(n=228)$ consideró estar capacitado para tratarlo una vez se desempeñe en un aula. Solo el 12,6\% $(n$ $=12,6 \%)$ señaló no estar capacitado. Si profundizamos en las respuestas obtenidas, en número de participantes que consignó tener una capacitación deficiente para trabajar el cambio climático en las aulas de educación primaria coincide con el 12,6\% $(n=33)$ de participantes que seńaló no estar lo suficientemente formado para impartir este tema. Por otra parte, el 37,5\% $(n=98)$ consideró que está lo suficientemente formado para tratarlo y el 49,8\% ( $n=130 \%)$ restante indicó que si bien está formado para tratar el tema, no lo está para hacerlo en profundidad, tal y como señala el C72: "Considero que estoy formada para abordar lo principal del tema, pero no para tratarlo con profundidad".

La formación en la universidad ha sido un tema recurrente en las respuestas obtenidas, ya que un número significativo de participantes mencionó que el cambio climático fue trabajado durante su formación universitaria. Ejemplo de esto se extrae de las siguientes unidades de información:

- C13: Sí, debido a todas las sesiones hipotéticas que se podrían dar en un aula de primaria que hemos recibido en la facultad de educación, aunque nunca se está capacitado del todo y siempre hay que estar en continua formación para mejorar.

- C109: Esta es una de las problemáticas que más se abordan en la universidad. Sin embargo, debemos llevarlo a la práctica, pues hoy en día existen muchos centros educativos que no tratan este tema tan relevante e importante. Es por ello que debemos animar, potenciar y formar a los futuros maestros para que entiendan su importancia y en un futuro lo lleven realmente a la práctica.

\section{Discusión y reflexiones finales}

La elección de profesorado en formación inicial de educación primaria para este estudio se debió a la importante labor que estos profesionales desempeńarán a lo largo de su labor en las aulas, tal como señalan Meira-Cartea y Arto-Blanco (2014).

Por otro lado, el alumnado universitario tiene una mayor afinidad con la cultura científica que otros grupos de población, además de la relevancia de la universidad como centro educativo y de innovación social.

En cuanto a los dos primeros objetivos del estudio - conocer lo que entiende el profesorado por cambio climático en formación inicial de educación primaria y, explorar sus conocimientos en torno a las causas, consecuencias y estrategias que se pueden llevar a cabo para mitigar el cambio climático-, los resultados de la investigación pusieron de manifiesto que el tratamiento de los problemas socioambientales sigue siendo uno de los retos actuales en las aulas escolares, por lo cual es necesario dotar al profesorado de las herramientas que le permitan el abordaje de estos contenidos.

El profesorado en formación inicial, en tanto, señaló las consecuencias más mediáticas, como el deshielo de los polos o el aumento del nivel del mar. Sin embargo, no seńaló consecuencias locales, como por ejemplo, la desertificación que se está produciendo en Andalucía (zona en dónde se ha realizado el estudio) y que impacta directamente en la productividad de la agricultura. Esto es significativo porque demuestra que el profesorado no termina de conocer cómo ciertas consecuencias del cambio climático pueden afectar su vida diaria, es decir, no es capaz de contextualizar la problemática. 
Además de lo anterior, los participantes del estudio presentaron concepciones alternativas relativas al cambio climático, entre ellas la mención a la capa de ozono (C17: "Los residuos perjudiciales que afectan a la capa de ozono producidos por el hombre, las fábricas, los desechos, etc.") o la de entender el aumento de $\mathrm{CO}_{2}$ como consecuencia del cambio climático, cuando esto en realidad corresponde a una causa. Ahora bien, entendemos que pueden referirse a la contaminación atmosférica que afecta a la salud, pero en ese sentido hay una cosa muy importante que debemos advertir: confundir la contaminación atmosférica con el cambio climático es igualmente preocupante, ya que se trata de dos problemas ambientales diferentes que comparten causas y por ello se suelen confundir, y aunque están interrelacionados (como casi todos los problemas ambientales), son cuestiones diferentes.

En relación con las estrategias pedagógicas para el abordaje de los contenidos relativos al cambio climático, el profesorado no hizo alusión a técnicas de adaptación, entre las que se cuentan, por ejemplo, aquellas tendientes a cambiar estilos de vida, sino que solo se enfocaron en la reducción del consumo. De igual modo, dicen que la vida cambiará debido al cambio climático y que es necesario intervenir, pero entre sus posibles estrategias no figura la resiliencia ciudadana (¿cómo cambiará para ellos y ellas?, ¿qué podrán hacer y qué no?), modificaciones en su alimentación (¿desaparecerán algunos alimentos? ¿se incluirán los insectos como parte de la dieta de las personas?), alteraciones en la gestión de las ciudades (creación de sitios de más sombra o más huertos urbanos para combatir la escasez de alimentos, etc.), o la adaptación individual para aprender a cultivar, obtener agua, etc., entre otros caminos de acción.

Respecto de las estrategias educativas, proponemos que el abordaje de las cuestiones socioambientales en el aula podría seguir el modelo constructivista; esto es, a partir de la selección concreta de temas socioambientales del entorno próximo del alumnado, se puede formular un problema e identificar sus causas y consecuencias, las condiciones a cambiar, así como las dificultades para que se produzca una transformación. Gracias a esta propuesta sería posible para el alumnado establecer prioridades para la participación y la realización de acciones sostenibles (Álvarez y Vega, 2009; Ferreras-Listán, Moreno-Fernández y Puig-Gutiérrez, 2019).

Así mismo, otra estrategia que bien podría llevarse a cabo sería la participación en debates específicos, esto es, la interacción con científicos (tomar por ejemplo los "cafés con ciencia" que se llevan a cabo desde diferentes instituciones universitarias en determinados momentos del curso académico). Ello permitiría el tratamiento de las concepciones erróneas, así como la ejecución de proyectos escolares y/o comunitarios que podrían abordarse desde una metodología de aprendizaje-servicio (Caballero, Domínguez, Miranda y Velo, 2018; Cebrián, Fernández, Fuertes, Moraleda y Segalàs, 2019; Monroe, Plate, Oxarart, Bowers \& Chaves, 2019), o bien, desde una perspectiva "glocal”2 (López-Fernández y Oller-Freixa, 2019; Moreno-Crespo y Moreno-Fernández, 2015; Morote, 2019).

Por tanto, consideramos que a pesar de estas carencias formativas, el profesorado en formación inicial de educación primaria consignó que el cambio climático es un tema prioritario que abordar en la escuela, y aunque los participantes evidenciaron una visión simplista de la problemática al confundir causas y consecuencias, en muchos casos ello puede deberse a que no contaron con una formación compleja respecto del tema, pese a que se consideraran a sí mismos debidamente capacitados para tratar el cambio climático, aunque fuera a nivel básico. Esto es un paso importante, ya que pone de manifiesto que estos profesionales están sensibilizados con la importancia de trabajar este tema.

Por último respecto del objetivo de determinar la relevancia que tiene el cambio climático como problemática socioambiental a tratar en educación primaria desde la perspectiva del futuro profesorado, los resultados de la investigación pusieron de manifiesto que la visión reduccionista del currículo genera que el cambio climático sea entendido también parcialmente, pese a tener este una arista natural y otra social, y que la mejor manera de abordar la temática sería desde dos ópticas conjuntas, con lo cual se gana una perspectiva más compleja y

2. Hace referencia a factores que inciden a nivel tanto de la dimensión global, como de la dimensión local, reuniendo características de ambas realidades en una misma dimensión: "Pensar globalmente y actuar localmente". 
holística del problema, acorde con las necesidades actuales del mundo en el que vivimos. Coincidimos así con Bustillo y Antón (2020) en "la necesidad de incorporar en la formación de los futuros docentes actuaciones que les permitan ser conscientes de sus limitaciones a la hora de interpretar temas complejos” (p. 207), así como con García-Pérez y De Alba (2008), en que

trabajar en torno a problemas sociales y ambientales relevantes en el marco de un currículo escolar de carácter integrado favorece el tratamiento de los graves problemas de nuestro tiempo y contribuye, por tanto, a la formación de los alumnos y alumnas como ciudadanos de nuestro mundo (p. 9).

Es en esta línea en la que creemos que es necesario seguir trabajando para profundizar acerca de las concepciones del profesorado en formación inicial o en formación permanente, respecto de las problemáticas socioambientales y su abordaje educativo con una perspectiva de ciudadanía planetaria y comprometida con su entorno (VarelaLosada, Arias-Correa y Vega-Marcote, 2019).

El artículo original fue recibido el 11 de mayo de 2020

El artículo revisado fue recibido el 10 de agosto de 2020

El artículo fue aceptado el 28 de agosto de 2020

\section{Referencias}

Álvarez, P. y Vega, P. (2009). Actitudes ambientales y conductas sostenibles. Implicaciones para la educación ambiental. Revista de Psicodidáctica, 14(2), 245-260. Recuperado de https://www.redalyc.org/pdf/175/17512724006.pdf

Azcárate, P., Navarrete, A., y García, E. (2012). Aproximación al nivel de inclusión de la sostenibilidad en los curricula universitarios. Profesorado, 16(2), 105-119. Recuperado de https://www.redalyc.org/pdf/567/56724395007.pdf

Aznar, P., Martínez-Agut, M. P., Palacios, B., Piñero, A., \& Ull, M. A. (2011). Introducing sustainability into university curricula: an indicator and baseline survey of the views of university teachers at the University of Valencia. Environmental Education Research, 17(2), 145-166. https://doi.org/10.1080/13504622.2010.502590

Bangay, C. \& Blum, N. (2010). Education responses to climate change and quality: Two parts of the same agenda? International Journal of Educational Development, 30(4), 359-368. https://doi.org/10.1016/j.ijedudev.2009.11.011

Barbosa, L. G., Lima, M. E., \& Machado, A. H. (2012). Controvérsias sobre o aquecimento global: Circulação de vozes e de sentidos produzidos em sala de aula. Ensaio Pesquisa em Educação em Ciências, 14(1), 113-130. https://doi.org/10.1590/1983-21172012140108

Barrón, A., Navarrete A., y Ferrer-Balas, D. (2010). Sostenibilización curricular en las universidades españolas. ¿̇Ha llegado la hora de actuar? Revista Eureka. Enseñanza y Divulgación de las Ciencias, 7, 297-315. Recuperado de https://revistas.uca.es/index.php/eureka/article/view/2657

Barros,H.C.L.yPinheiro,J.Q. (2013). Dimensiones psicológicas del calentamiento global conformelavisión deadolescentes brasileños. Estudos de Psicologia (Natal), 18(2), 173-182. https://doi.org/10.1590/S1413-294X2013000200002

Bustillo, J. y Antón, A. (2020). Análisis de la percepción que tiene el futuro profesorado sobre el estado actual del mundo. Revista Interuniversitaria de Formación del Profesorado, 34(1), 197-212. https://doi.org/10.47553/rifop.v34i1.75720

Caballero, P., Domínguez, G., Miranda, M. J., y Velo, C. (2018). Jornada de aventura "Superhéroes en la ecoescuela: una experiencia de aprendizaje servicio para conectar la universidad y el colegio. EmásF: Revista Digital de Educación Física, 9(54), 114-123. Recuperado de https://dialnet.unirioja.es/servlet/articulo?codigo=6571092

Cebrián, G., Fernández, M., Fuertes, M. T., Moraleda, A., y Segalàs, J. (2019). La influencia del aprendizaje-servicio en el desarrollo de competencias en sostenibilidad en estudiantes universitarios. Bordón. Revista de Pedagogía, 71(3), 151-167. https://doi.org/10.13042/bordon.2019.68276

Cohen, L. y Manion, L. (2002). Métodos de investigación educativa. Madrid: La Muralla. 
Escoz-Roldán, A., Gutiérrez-Pérez, J., Arto-Blanco, M., y Meira-Cartea, P. A. (2017). La representación social del cambio climático en el alumnado universitario español de Ciencias e Ingeniería. Enseñanza de las Ciencias: Revista de Investigación y Experiencias Didácticas, núm. extra, 1765-1770. Recuperado de https://ddd.uab.cat/record/184366

Ferreras-Listán, M., Moreno-Fernández, O., y Puig-Gutiérrez, M. (2019). Los itinerarios didácticos como recurso educativo para trabajar problemas socio-ambientales en el aula. En M. Ferreras-Listán, O. Moreno-Fernández, y M. Puig-Gutiérrez (Coord.), Innovación e investigación en la formación inicial del profesorado de infantil y primaria desde la didáctica de las ciencias experimentales y sociales (pp. 13-30). Barcelona: Octaedro.

García-Díaz, J. E. (2004). Educación ambiental, constructivismo y complejidad. Sevilla: Díada.

García-Pérez, F. F. y De Alba, N. (2008). ¿Puede la escuela del siglo XXI educar a los ciudadanos y ciudadanas del siglo XXI? Scripta Nova. Revista Electrónica de Geografía y Ciencias Sociales, 12(270) (122), 1-15. Recuperado de http://www.ub.edu/geocrit/sn/sn-270/sn-270-122.htm

García-Rodeja, I. y De Oliveira, L. G. (2012). Sobre el cambio climático y el cambio de los modelos de pensamiento de los alumnos. Enseñanza de las Ciencias: Revista de Investigación y Experiencias Didácticas, 30(3), 195-218. https://doi.org/10.5565/rev/ec/v30n3.695

Giddens, A. (2010). La política del cambio climático. Madrid: Alianza Editorial.

González-Gaudiano, E. y Meira-Cartea, P. A. (2020). Educación para el cambio climático. ¿Educar sobre el clima o para el cambio? Perfiles Educativos, 42(168), 157-174. https://doi.org/10.22201/iisue.24486167e.2020.168.59464

Grupo Intergubernamental de Expertos sobre el Cambio Climático, IPCC. (2013). Quinto Informe de Evaluación del IPCC. Cambio climático 2013. Bases fisicas. Informe del Grupo de trabajo I. Recuperado de https://www.ipcc.ch/site/assets/uploads/2018/03/WG1AR5_SPM_brochure_es.pdf

Grupo Intergubernamental de Expertos sobre el Cambio Climático, IPCC (2018). Calentamiento global de 1, $5^{\circ} \mathrm{C}$. Informe especial del IPCC sobre los impactos del calentamiento global de $1,5^{\circ} \mathrm{C}$ con respecto a los niveles preindustriales y las trayectorias correspondientes que deberian seguir las emisiones mundiales de gases de efecto invernadero, en el contexto del reforzamiento de la respuesta mundial a la amenaza del cambio climático, el desarrollo sostenible y los esfuerzos por erradicar la pobreza. Ginebra: Organización Meteorológica Mundial, OMM y Programa de Naciones Unidas para el Medio Ambiente, PNUMA.

Hess, D. J. \& Collins, B. M. (2018). Climate change and higher education: Assessing factors that affect curriculum requirements. Journal of Cleaner Production, 170, 1451-1458. https://doi.org/10.1016/j.jclepro.2017.09.215

Hess, D. J. \& Maki, A. (2019). Climate change belief, sustainability education, and political values: Assessing the need for higher-education curriculum reform. Journal of Cleaner Production, 228, 1157-1166. https://doi.org/10.1016/j.jclepro.2019.04.291

Karatzoglou, B. (2013). An in-depth literature review of the evolving roles and contributions of universities to education for sustainable development. Journal of Cleaner Production, 49, 44-53. https://doi.org/10.1016/j.jclepro.2012.07.043

Leal-Filho, W. (2010). Climate change at universities. Results of a world survey. En W. Leal-Filho (Ed.), Universities and climate change: Introducing climate change to university (pp. 1e19). Berlin: Springer.

Leal-Filho, W., Morgan, E. A., Godoy, E., Azeiteiro, U. M., Bacelar-Nicolau, P., Veiga-Ávila, L., Mac-Lean, C., \& Hugé, J. (2018). Implementing climate change research at universities: Barriers, potential and actions. Journal of Cleaner Production, 170, 269-277. https://doi.org/10.1016/j.jclepro.2017.09.105

Leiserowitz, A. (2004). Before and after The Day After Tomorrow: A U.S. study of climate change risk perception. Environment, 46(9), 22-37. https://climatecommunication.yale.edu/wp-content/uploads/2016/02/2004_11_ Before-and-after-The-Day-After-Tomorrow.pdf

Leiserowitz, A. (2007). Communicating the risks of global warming: American risk perceptions, affective images, and interpretive communities. En S. C. Moser \& L. Dilling (Eds.), Creating a climate for change. Communicating climate change and faciliting social change (pp. 44-63). Cambridge: Cambridge University Press.

Ley Orgánica 2/2006, de 3 de mayo, de Educación. Boletín Oficial del Estado, 4 de mayo de 2006, núm. 106. Recuperado de https://www.boe.es/buscar/pdf/2006/BOE-A-2006-7899-consolidado.pdf

López-Fernández, J. A. y Oller-Freixa, M. (2019). Los problemas medioambientales en la formación del profesorado de educación primaria. REIDICS. Revista de Investigación en Didáctica de las Ciencias Sociales, 4, 93-109. Recuperado de https://mascvuex.unex.es/revistas/index.php/reidics/article/download/2531-0968.04.93/2320/ 
McKenzie, M., Hart, P., Bai, H., \& Jickling, B. (2009). Fields of green: Restorying culture, environment, and education. Cresskill: Hampton Press Inc.

Meira-Cartea, P. A. (2013). La respuesta de la sociedad española antes el cambio climático. Madrid: Fundación Mapfrel Aldine Editorial.

Meira-Cartea, P. A., Gutiérrez-Pérez, J., Arto-Blanco, M., y Escoz-Roldán, A. (2018). Formación académica frente a cultura común en la alfabetización climática de estudiantes universitarios. Psyecology. Revista Bilingüe de Psicología Ambiental, 9(3), 301-340. https://doi.org/10.1080/21711976.2018.1483569

Meira-Cartea, P. A. y Arto-Blanco, M. (2014). Representaciones del cambio climático en estudiantes universitarios en España: aportes para la educación y la comunicación. Educar em Revista, 3, 15-33. https://doi.org/10.1590/0104-4060.38041

Mochizuki, Y. \& Bryan, A. (2015). Climate change education in the context of education for sustainable development: Rationale and principles. Journal of Education for Sustainable Development, 9(1), 4-26. https://doi.org/10.1177/0973408215569109

Molthan-Hill, P., Worsfold, N., Nagy, G. J., Leal-Filho, W., \& Mifsud, M. (2019). Climate change education for universities: A conceptual framework from an international study. Journal of Cleaner Production, 226, 1092-1101. https://doi.org/10.1016/j.jclepro.2019.04.053

Monroe, M. C., Plate, R. R., Oxarart, A., Bowers, A., \& Chaves, W.A. (2019). Identifying effective climate change education strategies: A systematic review of the research. Environmental Education Research, 25(6), 791-812. https://doi.org/10.1080/13504622.2017.1360842

Moreno-Crespo, P. y Moreno-Fernández, O. (2015). Problemas socioambientales: concepciones del profesorado en formación inicial. Andamios. Revista de Investigación Social, 12(29), 73-96. https://doi.org/10.29092/uacm.v12i29.20

Moreno-Fernández, O. y Navarro-Díaz, M. (2015). Educación ambiental, ciudadanía y participación. IJERI. International Journal of Educational Research and Innovation, 4, 175-186. Recuperado de https://www.upo.es/revistas/index. $\mathrm{php} / \mathrm{IJERI} /$ article/view/1470

Morote, A. F. (2019). La enseñanza del cambio climático en la educación primaria. Exploración a partir de las representaciones sociales del futuro profesorado y los manuales escolares de ciencias sociales. ENSAYOS, Revista de la Facultad de Educación de Albacete, 34(2), 213-228. Recuperado de https://www.revista.uclm.es/index.php/ensayos/article/view/2129

Organización de las Naciones Unidas, ONU. (1992). Convención Marco de las Naciones Unidas sobre cambio climático. Recuperado de https://www.acnur.org/fileadmin/Documentos/BDL/2009/6907.pdf

Organización de las Naciones Unidas, ONU. (2015). Convención Marco de las Naciones Unidas sobre cambio climático. Recuperado de https://unfccc.int/resource/docs/2015/cop21/spa/109s.pdf

Real Decreto 126/2014, de 28 de febrero, por el que se establece el currículo básico de la Educación Primaria. Boletín Oficial del Estado, 1 de marzo de 2014, núm. 52. Recuperado de https://www.boe.es/buscar/pdf/2014/BOE-A-2014-2222consolidado.pdf

Reis, J. \& Ballinger, R. C. (2020). Creating a climate for learning-experiences of educating existing and future decisionmakers about climate change. Marine Policy, 111, 103204. https://doi.org/10.1016/j.marpol.2018.07.007

Russell, C. (2016). On open access, the politics of citation, and generous scholarship. Canadian Journal of Environmental Education, 21, 5-12.

Schewe, J., Gosling, S., Reyer, Ch., Zhao, F., Ciais, P., Elliott, J., ... Warszawski, L. (2019). State-of-the-art global models underestimate impacts from climate extremes. Nature Communications, 10(1005). https://doi.org/10.1038/s41467-019-08745-6

Solís-Espallargas, C. y Valderrama-Hernández, R. (2015). La educación para la sostenibilidad en la formación de profesorado. ¿Qué estamos haciendo? Foro de Educación, 13(19), 165-192. http://dx.doi.org/10.14516/fde.2015.013.019.008

Sternäng, L. \& Lundholm, C. (2011). Climate change and morality: Students' perspectives on the individual and society. International Journal of Science Education, 33(8), 1131-1148. https://doi.org/10.1080/09500693.2010.503765

United Nations, UN. (2015). Transforming our world: the 2030 Agenda for Sustainable Development A/RES/70/1. Recuperado de https://sustainabledevelopment.un.org/post2015/transformingourworld

Varela-Losada, M., Arias-Correa, A., y Vega-Marcote, P. (2019). Educar para el cambio y la sostenibilidad: evaluación de una propuesta de aprendizaje experiencial para formar al profesorado en formación inicial. Revista Portuguesa de Educação, 32(2), 57-73. https://doi.org/10.21814/rpe.15303 